Pacific

Journal of

Mathematics

EXISTENCE OF TIME-PERIODIC SOLUTIONS TO THE NAVIER-STOKES EQUATIONS AROUND A MOVING BODY

Giovanni P. GALDi AND ANA L. SiLvestre

Volume $223 \quad$ No. 2

February 2006 


\title{
EXISTENCE OF TIME-PERIODIC SOLUTIONS TO THE NAVIER-STOKES EQUATIONS AROUND A MOVING BODY
}

\author{
Giovanni P. Galdi And Ana L. Silvestre
}

\begin{abstract}
We demonstrate the existence of time-periodic motions of an incompressible Navier-Stokes fluid subject to a time-periodic body force, occupying the region exterior to a body that performs a periodic rigid motion of same period.
\end{abstract}

\section{Introduction}

Consider a rigid body $\mathscr{B}$ moving through an infinitely extended Navier-Stokes liquid $\mathscr{L}$, which is subject to an external force $f$. If $\Omega$ is the three-dimensional region exterior to $\mathscr{B}$, with boundary $\Sigma$, the equations of motion of $\mathscr{L}$ with respect to a frame attached to $\mathscr{B}$ and with the origin at the center of mass of $\mathscr{B}$ are

$$
\left\{\begin{array}{rlrl}
\partial_{t} u & =v \Delta u-\nabla p+(V-u) \cdot \nabla u-\omega \times u+f \\
\nabla \cdot u & =0 & & \\
u & =V & & \text { on } \Sigma \times \mathbb{R}, \\
\lim _{|x| \rightarrow \infty} u(x, t)=0 & & \text { for } t \in \mathbb{R} ;
\end{array}\right.
$$

see [Galdi 2002]. Here $u=u(x, t)$ is the velocity field of the liquid, $v$ is the kinematic viscosity coefficient of $\mathscr{L}$, and $p=p(x, t)$ is the pressure field divided by the (constant) density of $\mathscr{L}$, and $\omega$ is the angular velocity of $\mathscr{B}$. The velocity field associated with the rigid motion of $\mathscr{B}$ is

$$
V(x, t)=\xi(t)+\omega(t) \times x,
$$

where $\xi$ is the velocity of the center of mass of $\mathscr{B}$.

The question we address is the following. Assume that $\mathscr{B}$ moves periodically with period $T$ (that is, $\xi$ and $\omega$ are periodic functions of time), and that $f$ is also periodic with the same period. Then, does the fluid execute a time-periodic motion

MSC2000: 76D03, 76D05, 35Q35.

Keywords: Navier-Stokes equations, time-periodic solutions, moving obstacle.

Galdi's was partially supported by NSF grants DMS-0103970 and DMS-0404834. Silvestre's work was partially supported by F.C.T. through POCTI-FEDER and projects POCTI/MAT/34735/99 and POCTI/MAT/61792/2004. 
of period $T$ ? Though simple in its formulation and physically significant, this problem seemingly has been solved only when $\mathscr{B}$ is at rest [Maremonti 1991a; 1991b; Kozono and Nakao 1996; Maremonti and Padula 1996; Salvi 1995; Yamazaki 2000; Galdi and Sohr 2004]. (See also [Morimoto 1971/72] and the references therein for the case where $\Omega$ is a bounded domain.) The methods adopted in all these papers do not extend directly to the case when $\mathscr{B}$ undergoes periodic motion; they basically revolve around the properties of solutions of the linearized problem, $\mathscr{P}_{L}$, obtained by disregarding the nonlinear term $u \cdot \nabla u$ in equation $(1-1)_{1}$. If the body is at rest, $\mathscr{P}_{L}$ involves only the Stokes operator, $A=-P \Delta$ (where $P$ is the Helmholtz projection), and it reduces to the well-known Stokes problem. If $\mathscr{B}$ is in motion, by contrast, $\mathscr{P}_{L}$ involves the linear operator

$$
A+(\xi+\omega \times x) \cdot \nabla u-\omega \times u .
$$

(In the appropriate function class, we have $P((\xi+\omega \times x) \cdot \nabla u-\omega \times u)=(\xi+$ $\omega \times x) \cdot \nabla u-\omega \times u$.) Then, especially due to the presence of the unbounded coefficient $\omega \times x$, the linearized problem is much more complicated than the Stokes problem and its functional properties, to date, are not completely understood; see [Hishida 1999; Galdi 2003; Farwig et al. 2004]. One must therefore resort to other approaches. Note that exactly the same difficulty arises in the study of the initialboundary and boundary value problems associated to (1-1), for whose results and corresponding methods we refer to [Hishida 1999; Galdi and Silvestre 2002; Galdi 2003; Silvestre 2004] and references therein.

To our knowledge, even for the simpler case when $\omega \equiv 0$ and $\xi \not \equiv 0$ no results are available.

In this paper we show the existence of weak and strong periodic solutions to problem (1-1) in the case when $\mathscr{B}$ moves by an arbitrary time-periodic motion and $f$ is time-periodic with the same period. We prove these results by means of the classical Faedo-Galerkin approach suitably coupled with an "invading domains" technique [Ladyzhenskaya 1969; Heywood 1980]. Specifically, in each bounded domain $\Omega_{k}$ of an increasing sequence of domains covering $\Omega$, we show the existence of a periodic solution $\left(u^{(k)}, p^{(k)}\right)$. This solution is "weak", in the sense of Leray and Hopf, for $\xi, \omega$ and $f$ of arbitrary size in a suitable function class, and for an arbitrary exterior domain $\Omega$. Moreover, if $\Omega$ is of class $C^{2}$ and the size of $\xi, \omega$ and $f$ is appropriately restricted, we prove the existence of more regular solutions such that $d u^{(k)} / d t, u^{(k)}, \nabla u^{(k)}, D^{2} u^{(k)} \in L^{2}\left(\Omega_{k} \times[0, T]\right)$, and $p^{(k)}, \nabla p^{(k)} \in L^{2}\left(\Omega_{k} \times[0, T]\right)$. Because the term $(\xi+\omega \times x) \cdot \nabla u^{(k)}-\omega \times u^{(k)}$ possesses nice functional properties on each bounded $\Omega_{k}$ [Galdi and Silvestre 2002; 2005; Silvestre 2004], we are able to obtain estimates for $u^{(k)}$, uniformly in $k$, that allow us to pass to the limit $k \rightarrow \infty$ and to prove that weak (Theorem 3.2) and strong (Theorem 4.1) periodic solutions to (1-1) exist in the whole of $\Omega$. In the 
special case $\xi \equiv \omega \equiv 0$, our results improve those previously known, in that we require no regularity on $\Omega$ (versus the $C^{2}$-regularity needed in [Maremonti and Padula 1996]) in the case of weak solutions, and only $C^{2}$-regularity (versus the $C^{3}$-regularity needed in [Salvi 1995]) in the case of strong solutions.

The uniqueness problem is left open, even for strong solutions. As shown in [Galdi and Sohr 2004] for the simpler instance $\xi \equiv \omega \equiv 0$, uniqueness is not related to the local regularity of solutions but, rather, to their asymptotic behavior in space. The determination of this latter for the case at hand appears to be a challenging question that will be treated elsewhere.

The paper is organized as follows. After recalling in Section 2 some notation and preparatory results, in Section 3 we show the existence of weak periodic solution, while Section 4 is dedicated to the existence of strong periodic solutions.

\section{Notation and preparatory results}

Let $\mathscr{A}$ be a domain of $\mathbb{R}^{3}$. We denote by $\delta(\mathscr{A})$ the diameter of $\mathscr{A}$ and, for $R>\delta(\mathscr{A})$, we set $\mathscr{A}_{R}=\mathscr{A} \cap B_{R}$ and $\mathscr{A}^{R}=\mathscr{A} \backslash \overline{A_{R}}$, where $B_{R}=\left\{x \in \mathbb{R}^{3}:|x|<R\right\}$, and the bar denotes closure.

An exterior domain is the complement of the closure of a bounded domain in $\mathbb{R}^{3}$.

We shall use standard notation for function spaces [Adams 1975]. For instance, $L^{q}(\mathscr{A}), H^{m}(\mathscr{A}):=W^{m, 2}(\mathscr{A}), H_{0}^{m}(\mathscr{A}):=W_{0}^{m, q}(\mathscr{A})$, etc., denote the usual Lebesgue and Sobolev spaces on the domain $\mathscr{A}$, with norms $\|\cdot\|_{q, \mathscr{A}}$ and $\|\cdot\|_{m, 2, \mathscr{A}}$, respectively.

If $G, H$ are second-order tensor fields and $g, h$ are vector fields on $\mathscr{A}$, we set

$$
(G, H)_{\mathscr{A}}=\int_{\mathscr{A}} G_{i j} H_{i j}, \quad(g, h)_{\mathscr{A}}=\int_{\mathscr{A}} g_{i} h_{i},
$$

whenever the integrals make sense. If there is no danger of confusion, we shall omit the subscript $\mathscr{A}$.

The trace space on $\partial \mathscr{A}$ for functions from $H^{m}(\mathscr{A})$ is denoted by $H^{m-1 / 2}(\partial \mathscr{A})$ and its norm by $\|\cdot\|_{m-1 / 2, \partial \mathscr{A}}$. Classical properties and results related to these spaces can be found in [Adams 1975; Galdi 1994a]. The following spaces of solenoidal functions will be needed:

$$
\begin{aligned}
& \mathscr{D}(\mathscr{A})=\left\{\phi \in C_{0}^{\infty}(\mathscr{A}): \nabla \cdot \phi=0\right\}, \\
& H(\mathscr{A})=\text { completion of } \mathscr{D}(\mathscr{A}) \text { in the norm }\|\cdot\|_{2}, \\
& V(\mathscr{A})=\text { completion of } \mathscr{D}(\mathscr{A}) \text { in the norm }\|\nabla(\cdot)\|_{2} .
\end{aligned}
$$

The dual space of $V(\mathscr{A})$ will be denoted by $V^{\prime}(\mathscr{A})$ with norm $\|\cdot\|_{-1, \mathscr{A}}$ and the duality $\langle F, u\rangle_{\mathscr{A}}$ will indicate the value of $F \in V^{\prime}(\mathscr{A})$ at $u \in V(\mathscr{A})$. If $\mathscr{A}$ is locally 
Lipschitzian with outward unit normal $N$, we have

$$
\begin{aligned}
H(\mathscr{A}) & =\left\{\Phi \in L^{2}(\mathscr{A}): \nabla \cdot \Phi=0 \text { and } \Phi_{\mid \partial \mathscr{A}} \cdot N=0\right\}, \\
V(\mathscr{A}) & =\left\{\Phi \in H_{l o c}^{1}(\overline{\mathscr{A}}): \nabla \Phi \in L^{2}(\mathscr{A}), \nabla \cdot \Phi=0 \text { and } \Phi_{\mid \partial \mathscr{A}}=0\right\} ;
\end{aligned}
$$

see [Temam 1984; Galdi 1994a]. The orthogonal complement of $H(\mathscr{A})$ in $L^{2}(\mathscr{A})$ is

$$
G(\mathscr{A})=\left\{\nabla \pi \in L^{2}(\mathscr{A}): \pi \in H_{l o c}^{1}(\overline{\mathscr{A}})\right\} .
$$

The orthogonal projection of $L^{2}(\mathscr{A})$ onto $H(\mathscr{A})$ is denoted by $P$.

If $X$ is a Banach space, we denote by $L^{q}(0, T ; X)$ the space of all measurable functions from $[0, T]$ to $X$, such that $\int_{0}^{T}\|u(t)\|_{X}^{p} d t<\infty$, and by $C([0, T] ; X)$ the space of continuous function from $[0, T]$ to $X$.

Lemma 2.1. Let $X_{0}, X_{1}, X$ be Hilbert spaces such that the injection of $X_{0}$ into $X$ is compact and the injection of $X$ into $X_{1}$ is continuous. Then the injection of the space

$$
\left\{v \in L^{2}\left(0, T ; X_{0}\right): \frac{d v}{d t} \in L^{1}\left(0, T ; X_{1}\right)\right\} .
$$

into $L^{2}(0, T ; X)$ is compact.

Proof. See [Temam 1984].

The boundary velocity $u(x, t)=\xi(t)+\omega(t) \times x$ has a solenoidal extension:

Lemma 2.2. Let $\Omega$ be an exterior domain of $\mathbb{R}^{3}$, and let $\xi, \omega \in H^{1}(0, T)$. Given $\epsilon>0$, there exists a solenoidal function $\tilde{u} \in H^{1}\left(0, T ; W^{m, q}(\Omega)\right), m \in \mathbb{N}, q \in[1, \infty]$, such that

$$
\begin{aligned}
& \|\tilde{u}\|_{H^{1}\left(0, T ; W^{m, q}(\Omega)\right)} \leq C(\Sigma, m, q)\left(\|\xi\|_{H^{1}(0, T)}+\|\omega\|_{H^{1}(0, T)}\right) \\
& \|\tilde{u}(t)\|_{m, q} \leq C(\Sigma, T, m, q)\left(\|\xi\|_{H^{1}(0, T)}+\|\omega\|_{H^{1}(0, T)}\right) \quad \text { for all } t \in[0, T] .
\end{aligned}
$$

Moreover,

$$
\left|\int_{\Omega_{R}} v(x, t) \cdot \nabla \tilde{u}(x, t) \cdot v(x, t) d x\right|<\epsilon\|\nabla v(t)\|_{2}^{2},
$$

for all $v \in C\left([0, T] ; V\left(\Omega_{R}\right)\right), t \in[0, T], R>\delta(\mathscr{B})$, and $\tilde{u}$ is $T$-periodic if $\xi$ and $\omega$ are $T$-periodic.

Proof. Using Lemma [Galdi 1994a, III.6.2], we consider a function $\eta_{\alpha} \in C_{0}^{\infty}(\bar{\Omega})$ such that $0 \leq \eta_{\alpha} \leq 1, \eta_{\alpha}=1$ if $\operatorname{dist}(x, \Sigma)<e^{-1 / \alpha} / 2, \eta_{\alpha}=0$ if $\operatorname{dist}(x, \Sigma) \geq 2 e^{-1 / \alpha}$, and $\left|\nabla \eta_{\alpha}(x)\right| \leq \alpha \operatorname{dist}(x, \Sigma)$, for all $x \in \Omega$, with $\alpha>0$. The extension is defined by

$$
\tilde{u}(x, t)=-\nabla \times\left(\eta_{\alpha}(x)\left(\xi_{i}(t) x_{(i+1) \bmod 3} e_{i}+\frac{1}{2}|x|^{2} \omega(t)\right)\right) .
$$

Taking into account the properties of the function $\eta_{\alpha}$ [Galdi 1994b, Chapter IX], it is possible to choose $\alpha$ such that $\tilde{u}$ satisfies the desired properties for a given $\epsilon$. 
We end this section with some fundamental estimates of suitable three-linear forms.

Lemma 2.3. Let $\Omega$ be an exterior domain of $\mathbb{R}^{3}$, and let $v \in V\left(\Omega_{R}\right) \cap H^{2}\left(\Omega_{R}\right)$, $\omega \in H^{1}(0, T)$. Then, for any $\varepsilon>0$ there is $C=C(\Omega, \varepsilon)>0$ such that

(i) $(v \cdot \nabla v, P \Delta v)_{\Omega_{R}} \leq C\left(\|\nabla v\|_{2, \Omega_{R}}^{4}+\|\nabla v\|_{2, \Omega_{R}}^{6}\right)+\varepsilon\|P \Delta v\|_{2, \Omega_{R}}^{2}$ and

(ii) $((\omega \times v-\omega \times x \cdot \nabla v), P \Delta v)_{\Omega_{R}} \leq C\left(\|\omega\|_{H^{1}(0, T)}+\|\omega\|_{H^{1}(0, T)}^{2}\right)\|\nabla v\|_{2, \Omega_{R}}^{2}+$ $\varepsilon\|P \Delta v\|_{2, \Omega_{R}}^{2}$.

Proof. The inequality in (i) is well known; see [Heywood 1980], for example. The proof of (ii) is given in [Galdi and Silvestre 2005].

\section{Existence of periodic weak solutions}

Denote by $\mathscr{D}_{T, p}$ the class of functions $\Phi$ that are infinitely differentiable in $\Omega \times$ $[0, T]$, of compact support in $\Omega$, and satisfying $\operatorname{div} \Phi(x, t)=0$ in $\Omega \times[0, T]$ and $\Phi(x, 0)=\Phi(x, T)$ in $\Omega$. If we formally multiply through both sides of $(1-1)_{1}$ by $\Phi \in \mathscr{D}_{T, p}$ and integrate by parts over $\Omega \times[0, T]$, we obtain

$$
\begin{aligned}
(u(T)- & u(0), \Phi(0)) \\
& =\int_{0}^{T}\left(\left(u, \Phi_{t}\right)-v(\nabla u, \nabla \Phi)+((V-u) \cdot \nabla u, \Phi)-(\omega \times u, \Phi)+\langle f, \Phi\rangle\right) .
\end{aligned}
$$

Thus, if $u$ is time-periodic of period $T$, the right-hand side of this equation vanishes. Conversely, if $u$ is a sufficiently regular field (in space and time) for which the righthand side of the relation above vanishes for all $\Phi \in \mathscr{D}_{T, P}$, it follows by standard arguments that $u$ satisfies $(1-1)_{1,2}$ for some pressure field $p$ and that $u(0)=u(T)$. We are thus led to:

Definition 3.1. A vector field $u$ is a periodic weak solution to (1-1) if

(i) $u-\tilde{u} \in L^{2}(0, T ; V(\Omega))$, where $\tilde{u}$ is the extension given in Lemma 2.2;

(ii) for all $\Phi \in \mathscr{D}_{T, p}$,

(3-1) $\int_{0}^{T}\left(\left(u, \Phi_{t}\right)-v(\nabla u, \nabla \Phi)+((V-u) \cdot \nabla u, \Phi)-(\omega \times u, \Phi)+\langle f, \Phi\rangle\right)=0$.

Remark. It is easy to show that, if $f \in L^{1}\left(0, T ; V^{\prime}(\Omega)\right)$ and $\xi, \omega \in H^{1}(0, T)$, every periodic weak solution satisfies

$$
\frac{d u}{d t} \in L^{1}\left(0, T ; V^{\prime}\left(\Omega_{R}\right)\right) \text { for all } R>\delta(\mathscr{B}),
$$

and so, in particular,

$$
u \in C\left([0, T] ; V^{\prime}\left(\Omega_{R}\right)\right) \quad \text { for all } R>\delta(\mathscr{B}) .
$$


In fact, set $\Phi=\varphi \psi$ in (3-1), where $\varphi \in \mathscr{D}(\Omega)$ and $\psi \in C_{0}^{\infty}(0, T)$. We obtain

$$
\int_{0}^{T}(u(t), \varphi) \psi^{\prime}(t)=-\int_{0}^{T} G_{\varphi}(t) \psi(t) \quad \text { for all } \psi \in C_{0}^{\infty}(0, T),
$$

where

$$
G_{\varphi}(t)=-v(\nabla u, \nabla \varphi)+((V-u) \cdot \nabla u, \varphi)-(\omega \times u, \varphi)+\langle f, \varphi\rangle .
$$

Using the inequality

$$
\|u\|_{2, \Omega_{R}} \leq C\left(\Omega_{R}\right)\left(\|\nabla u\|_{2}+\|\tilde{u}\|_{1,2}\right),
$$

along with the assumption on $f$, we obtain for a.a. $t \in[0, T]$

$$
\begin{aligned}
& \left|G_{\varphi}(t)\right| \leq C\left(\Omega_{R}, v\right) \\
& \times\left(\left(1+\|\xi\|_{H^{1}(0, T)}+\|\omega\|_{H^{1}(0, T)}\right)\|\nabla u\|_{2, \Omega_{R}}+\left\|\nabla u_{2}\right\|_{2}^{2}+\|\tilde{u}\|_{1,2}^{2}+\|f\|_{-1}\right)\|\varphi\|_{1,2, \Omega_{R}} .
\end{aligned}
$$

Thus, with the help of Definition 3.1(ii), we find $G_{\varphi}(t)=\langle g(t), \varphi\rangle$ with $g \in$ $L^{1}\left(0, T ; V^{\prime}\left(\Omega_{R}\right)\right)$ and

$$
\frac{d}{d t}(u, \varphi)=\langle g, \varphi\rangle
$$

in the sense of distributions on $[0, T]$. The desired property is then proved.

The objective of this section is to show:

Theorem 3.2. Let $\Omega$ be an exterior domain of $\mathbb{R}^{3}$. Let $\xi, \omega \in H^{1}(0, T)$ with $\xi(0)=\xi(T), \omega(0)=\omega(T)$, and let $f \in L^{2}\left(0, T ; V^{\prime}(\Omega)\right)$ be periodic in time of period $T$. Then there exists at least one periodic weak solution to (1-1).

For the proof we use the "invading domains" procedure (see [Heywood 1980], for instance), in two steps. In the first, using the method of [Prouse 1963], we show the existence of a periodic weak solution $u_{m}$ on bounded domains $\Omega_{R_{m}}$, $R_{m}>\delta(\mathscr{B}), m \in \mathbb{N}$, and establish suitable a priori estimates. In the second step, we let $R_{m} \rightarrow \infty$ and show that $u_{m}$ converges, in a suitable sense, to a periodic weak solution to $(1-1)$.

Step 1: Construction of approximating periodic weak solutions in $\boldsymbol{\Omega}_{\boldsymbol{R}_{m}}$. Let $\mathscr{Y}=$ $\left\{R_{m}: m \in \mathbb{N}\right\}$ be an increasing and unbounded sequence of positive numbers with $R_{1}>\delta(\mathscr{B})$ and let $\left\{\Omega_{R}: R \in \mathscr{Y}\right\}$ with $\bigcup_{R \in \mathscr{S}} \Omega_{R}=\Omega$ be the corresponding sequence of bounded domains covering $\Omega$.

In each $\Omega_{R}, R \in \mathscr{Y}$, we shall look for a $T$-periodic solution $u_{R}$ in the form $u_{R}=v_{R}+\tilde{u}$, with $\tilde{u}$ given by Lemma 2.2, and $v_{R}$ (in the appropriate functional 
class) satisfying the identity

$$
\begin{array}{r}
\frac{d}{d t}\left(v_{R}, \varphi\right)_{\Omega_{R}}=-v\left(\nabla v_{R}, \nabla \varphi\right)_{\Omega_{R}}+\left(\left(V-v_{R}\right) \cdot \nabla v_{R}, \varphi\right)_{\Omega_{R}}-\left(v_{R} \cdot \nabla \tilde{u}, \varphi\right)_{\Omega_{R}} \\
-\left(\tilde{u} \cdot \nabla v_{R}, \varphi\right)_{\Omega_{R}}-\left(\omega \times v_{R}, \varphi\right)_{\Omega_{R}}+\langle\tilde{f}, \varphi\rangle_{\Omega_{R}}
\end{array}
$$

for all $\varphi \in \mathscr{D}\left(\Omega_{R}\right)$, and a.a. $\left.t \in\right] 0, T[$, where

$$
\tilde{f}=f+v \Delta \tilde{u}-\partial_{t} \tilde{u}-\tilde{u} \cdot \nabla \tilde{u}+V \cdot \nabla \tilde{u}-\omega \times \tilde{u} .
$$

Under the hypotheses of Theorem 3.2 and with the help of Lemma 2.2, we deduce that $\tilde{f}$ is periodic of period $T, \tilde{f} \in L^{2}\left(0, T ; V^{\prime}(\Omega)\right)$, and that

$$
\begin{aligned}
\|\tilde{f}\|_{L^{2}\left(0, T ; V^{\prime}\left(\Omega_{R}\right)\right)} & \leq\|\tilde{f}\|_{L^{2}\left(0, T ; V^{\prime}(\Omega)\right)} \\
& \leq C(\Sigma)\left(\|\xi\|_{H^{1}(0, T)}+\|\omega\|_{H^{1}(0, T)}\right)+\|f\|_{L^{2}\left(0, T ; V^{\prime}(\Omega)\right)} .
\end{aligned}
$$

For each $R \in \mathscr{Y}$, we consider a base $\left\{w_{R i}\right\}_{i \in \mathbb{N}}$ of $V\left(\Omega_{R}\right)$ orthonormal in $H\left(\Omega_{R}\right)$. We let

$$
v_{R k}(x, t)=\sum_{i=1}^{k} c_{R k i}(t) w_{R i}(x),
$$

where the coefficients $c_{R k}=\left\{c_{R k 1}, \ldots, c_{R k k}\right\}$ are required to solve the system of ordinary differential equations

$$
\frac{d c_{R k j}}{d t}=\sum_{i=1}^{k} A_{i j}(t) c_{R k i}+\sum_{i, l=1}^{k} B_{i l j} c_{R k i} c_{R k l}+C_{j}(t), \quad j=1, \ldots, k,
$$

where

$$
\begin{array}{rlr}
A_{i j} & =-v\left(\nabla w_{R i}, \nabla w_{R j}\right)_{\Omega_{R}}-\left(\omega \times w_{R i}, w_{R j}\right)_{\Omega_{R}}+\left(V \cdot \nabla w_{R i}, w_{R j}\right)_{\Omega_{R}} \\
B_{i l j} & =-\left(w_{R i} \cdot \nabla w_{R l}, w_{R j}\right)_{\Omega_{R}}, & -\left(w_{R i} \cdot \nabla \tilde{u}, w_{R j}\right)_{\Omega_{R}}-\left(\tilde{u} \cdot \nabla w_{R i}, w_{R j}\right)_{\Omega_{R}}, \\
C_{j} & =\left\langle\tilde{f}, w_{R j}\right\rangle_{\Omega_{R}} .
\end{array}
$$

Following [Prouse 1963], we begin to show the existence of a $T$-periodic solution to the system (3-5).

Lemma 3.1. System (3-5) has a solution $c_{R k} \in H^{1}(0, T)$, such that $c_{R k}(0)=$ $c_{R k}(T)$.

Proof. For each $R \in \mathscr{S}$ and each $k \in \mathbb{N}$, we choose an initial velocity $v_{0 R k} \in$ $\operatorname{span}\left\{w_{R 1}, \ldots, w_{R k}\right\}$ and set $c_{R k j}(0)=c_{0 R k j}:=\left(w_{R j}, v_{0 R k}\right)_{\Omega_{R}}$. Since $\xi, \omega \in$ $H^{1}(0, T)$ and $f \in L^{2}\left(0, T, V^{\prime}(\Omega)\right)$, the system (3-5) has a unique solution $c_{R k} \in$ $H^{1}\left(0, T_{R k}\right)$ for some $T_{R k} \leq T$. Multiplying (3-5) by $c_{R k j}$, summing over $j$, integrating by parts, and recalling that

$$
\left(\left(V_{R k}-v_{R k}\right) \cdot \nabla v_{R k}, v_{R k}\right)=\left(\omega \times v_{R k}, v_{R k}\right)=\left(\tilde{u} \cdot \nabla v_{R k}, v_{R k}\right)=0,
$$


(where we have omit the subscript $\Omega_{R}$ for simplicity), we see that $v_{R k}$ satisfies the equation

$$
\frac{1}{2} \frac{d}{d t}\left\|v_{R k}\right\|_{2}^{2}+v\left\|\nabla v_{R k}\right\|_{2}^{2}=\left\langle\tilde{f}, v_{R k}\right\rangle-\left(v_{R k} \cdot \nabla \tilde{u}, v_{R k}\right) .
$$

Using Lemma 2.2 with $\epsilon=\frac{1}{4} v$, we get

$$
-\left(v_{R k} \cdot \nabla \tilde{u}, v_{R k}\right) \leq \frac{1}{4} v\left\|\nabla v_{R k}\right\|_{2}^{2}
$$

and since

$$
\left\langle\tilde{f}, v_{R k}\right\rangle_{\Omega_{R}} \leq\|\tilde{f}\|_{-1}\left\|\nabla v_{R k}\right\|_{2} \leq \frac{1}{4} v\left\|\nabla v_{R k}\right\|_{2}^{2}+C(v)\|\tilde{f}\|_{-1}^{2},
$$

we obtain

$$
\frac{d}{d t}\left\|v_{R k}\right\|_{2}^{2}+v\left\|\nabla v_{R k}\right\|_{2}^{2} \leq C(v)\|\tilde{f}\|_{-1}^{2} .
$$

Using the Poincaré inequality

$$
\|\nabla w\|_{2} \geq \frac{C}{R}\|w\|_{2}, \quad w \in H_{0}^{1}\left(\Omega_{R}\right),
$$

with $C$ a positive, absolute constant, we get

$$
\frac{d}{d t}\left\|v_{R k}\right\|_{2}^{2}+\frac{v C_{1}}{R^{2}}\left\|v_{R k}\right\|_{2}^{2} \leq C_{2}(v)\|\tilde{f}\|_{-1}^{2} .
$$

Consequently,

$$
e^{\nu C_{1} t / R^{2}}\left\|v_{R k}(t)\right\|_{2}^{2} \leq\left\|v_{0 R k}\right\|_{2}^{2}+C_{2}(\nu) \int_{0}^{T_{R k}} e^{\nu C_{1} \tau / R^{2}}\|\tilde{f}(\tau)\|_{-1}^{2} d \tau
$$

for all $t \in\left[0, T_{R k}\right]$. From this inequality it follows that

$$
\left\|v_{R k}(t)\right\|_{2}^{2} \leq\left\|v_{0 R k}\right\|_{2}^{2}+C_{2}(v) \int_{0}^{T} e^{\nu C_{1} \tau / R^{2}}\|f(\tau)\|_{-1}^{2} d \tau
$$

for all $t \in\left[0, T_{R k}\right]$. Using the orthogonality properties of $\left\{w_{R 1}, \ldots, w_{R k}\right\}$ we have $\left|c_{R k}(t)\right|=\left\|v_{R k}(t)\right\|_{2}$, from which we conclude that $T_{R k}=T$.

Let $\varrho$ be such that

$$
\varrho^{2} \geq \frac{C_{2}(\nu) \int_{0}^{T} e^{\nu C_{1} \tau / R^{2}}\|\tilde{f}(\tau)\|_{-1}^{2} d \tau}{1-e^{-\nu C_{1} T / R^{2}}}
$$

and let $\mathbb{B}_{\varrho}^{k}$ be the ball of radius $\varrho$ in $\mathbb{R}^{k}$. In view of (3-10) and (3-12), if $\left|c_{R k}(0)\right|=$ $\left\|v_{0 R k}\right\|_{2} \leq \varrho$ then

$$
\left|c_{R k}(T)\right|=\left\|v_{R k}(T)\right\|_{2} \leq \varrho,
$$

and thus the map $\mathscr{T}: \mathbb{B}_{\varrho}^{k} \rightarrow \mathbb{B}_{\varrho}^{k}$ such that $\mathscr{T}\left(c_{0 R k}\right)=c_{R k}(T)$ is well defined. By the same procedure used in [Prouse 1963], we can show that the map $\mathscr{T}$ is continuous, 
and therefore has a fixed point; that is, there exists a solution to (3-5) such that $c_{R k}(0)=c_{R k}(T)$.

Here are some useful estimates for the approximating solution in $\Omega_{R}$.

Lemma 3.2. There exists a positive constant $C=C(v, \Sigma)$ such that

(3-13) $\quad \int_{0}^{T}\left\|\nabla v_{R k}(\tau)\right\|_{2}^{2} d \tau \leq C\left(\|f\|_{L^{2}\left(0, T ; V^{\prime}(\Omega)\right)}^{2}+\|\xi\|_{H^{1}(0, T)}^{2}+\|\omega\|_{H^{1}(0, T)}^{2}\right)$.

Moreover, there exists a positive constant $C$ independent of $k \in \mathbb{N}$ such that

$$
\left\|v_{R k}(t)\right\|_{2} \leq C \quad \text { for all } t \in[0, T] \text { and all } k \in \mathbb{N} \text {. }
$$

Proof. Since, by Lemma 3.1, $v_{R k}$ is $T$-periodic, integrating (3-8) over $[0, T]$ we find

$$
\int_{0}^{T}\left\|\nabla v_{R k}(\tau)\right\|_{2}^{2} d \tau \leq C(v) \int_{0}^{T}\|\tilde{f}(\tau)\|_{-1}^{2} d \tau
$$

and then we use (3-4). This proves (3-13).

Equation (3-14) is an immediate consequence of (3-11).

We can now easily show the existence of a periodic weak solution on each $\Omega_{R}$, for $R \in \mathscr{Y}$. Actually, using Lemma 3.2 and well-known procedures (see [Galdi 2000], for example), we prove the existence of a field $v_{R}$ and of a subsequence, again denoted by $\left\{v_{R k}\right\}_{k \in \mathbb{N}}$, such that

$$
\begin{aligned}
& v_{R} \in L^{2}\left(0, T ; V\left(\Omega_{R}\right)\right) \cap L^{\infty}\left(0, T ; H\left(\Omega_{R}\right)\right), \\
& v_{R k} \rightarrow v_{R} \text { weakly in } L^{2}\left(0, T ; V\left(\Omega_{R}\right)\right), \\
& v_{R k} \rightarrow v_{R} \text { strongly in } L^{2}\left(0, T ; H\left(\Omega_{R}\right)\right), \\
& v_{R k}(t) \rightarrow v_{R}(t) \text { weakly in } L^{2}\left(\Omega_{R}\right), \text { for all } t \in[0, T] .
\end{aligned}
$$

Recalling that $v_{R k}(0)=v_{R k}(T)$, for $k \in \mathbb{N}$, the last condition in (3-16) implies that $v_{R}(0)=v_{R}(T)$, namely, that $v_{R}$ is $T$-periodic. Moreover, in view of (3-16) 2 and of (3-13), we find

$$
\text { (3-17) } \int_{0}^{T}\left\|\nabla v_{R}(\tau)\right\|_{2, \Omega_{R}}^{2} d \tau \leq C\left(\|f\|_{L^{2}\left(0, T ; V^{\prime}(\Omega)\right)}^{2}+\|\xi\|_{H^{1}(0, T)}^{2}+\|\omega\|_{H^{1}(0, T)}^{2}\right),
$$

with $C=C(v, \Omega)>0$. Finally, coupling (3-16) with classical arguments, we can prove that, for all $R \in \mathscr{Y}, v_{R}$ satisfies condition (3-2). Using (3-16) $)_{1}$ along with a standard procedure (see [Temam 1984], for instance), we can also show that the right-hand side of (3-2), with $v \equiv v_{R}$, defines a continuous (linear) functional on $V\left(\Omega_{R}\right)$, and that

$$
\left.\frac{d}{d t}\left(v_{R}, \varphi\right)_{\Omega_{R}}=\left\langle\frac{d v_{R}}{d t}, \varphi\right\rangle_{\Omega_{R}} \quad \text { for all } \varphi \in V\left(\Omega_{R}\right) \text { and a.a. } t \in\right] 0, T[,
$$


where

$$
\frac{d v_{R}}{d t} \in L^{4 / 3}\left(0, T ; V^{\prime}\left(\Omega_{R}\right)\right) .
$$

Consequently, from (3-2) we deduce that

$$
\begin{array}{r}
\left\langle\frac{d v_{R}}{d t}, \varphi\right\rangle_{\Omega_{R}}=-v\left(\nabla v_{R}, \nabla \varphi\right)_{\Omega_{R}}+\left(\left(V-v_{R}\right) \cdot \nabla v_{R}, \varphi\right)_{\Omega_{R}}-\left(v_{R} \cdot \nabla \tilde{u}, \varphi\right)_{\Omega_{R}} \\
-\left(\tilde{u} \cdot \nabla v_{R}, \varphi\right)_{\Omega_{R}}-\left(\omega \times v_{R} \cdot \varphi\right)_{\Omega_{R}}+\langle\tilde{f}, \varphi\rangle_{\Omega_{R}}
\end{array}
$$

for all $\varphi \in V\left(\Omega_{R}\right)$ and a.a. $\left.t \in\right] 0, T[$.

Step 2: Convergence of the sequence $\left\{v_{R}+\tilde{u}\right\}_{R \in \mathscr{S}}$ to a periodic weak solution to (1-1). We extend $v_{R}$ by zero outside $\Omega_{R}$, for $R \in \mathscr{Y}$, and continue to denote the extension by $v_{R}$. Clearly, the extended fields satisfy (3-17) and (3-18). We shall next prove some appropriate estimates for them.

Lemma 3.3. Let $R_{0}>\delta(\mathscr{B})$. There exists a positive constant $C$ depending only on the data and $R_{0}$ such that

$$
\int_{0}^{T}\left\|v_{R}(t)\right\|_{2, \Omega_{R_{0}}}^{2} d t+\int_{0}^{T}\left\|\frac{d v_{R}}{d t}(t)\right\|_{V^{\prime}\left(\Omega_{R_{0}}\right)} d t \leq C,
$$

for all $R>R_{0}, R \in \mathscr{Y}$.

Proof. The estimate on $v_{R}$ is an obvious consequence of (3-9) and of (3-17). Let $\varphi$ be any function in $\mathscr{D}\left(\Omega_{R_{0}}\right)$. From Hölder's inequality, Lemma 2.2, (3-9) and the Sobolev inequalities

$$
\|w\|_{6} \leq C\|\nabla w\|_{2}, \quad\|w\|_{3} \leq C R\|\nabla w\|_{2}, \quad w \in V\left(\Omega_{R}\right)
$$

with $C$ a positive, absolute constant, we find

$$
\begin{aligned}
-\left(\nabla v_{R}, \nabla \varphi\right) & \leq\left\|\nabla v_{R}\right\|_{2}\|\nabla \varphi\|_{2}, \\
\left(V \cdot \nabla v_{R}, \varphi\right) & \leq\|V\|_{3, \Omega_{R_{0}}}\left\|\nabla v_{R}\right\|_{2}\|\varphi\|_{6} \\
& \leq C(\Sigma, T)\left(R_{0}\|\xi\|_{H^{1}(0, T)}+R_{0}^{2}\|\omega\|_{H^{1}(0, T)}\right)\left\|\nabla v_{R}\right\|_{2}\|\nabla \varphi\|_{2}, \\
-\left(v_{R} \cdot \nabla v_{R}, \nabla \varphi\right) & \leq\left\|v_{R}\right\|_{6}\left\|\nabla v_{R}\right\|_{2}\|\varphi\|_{3} \leq C R_{0}\left\|\nabla v_{R}\right\|_{2}^{2}\|\nabla \varphi\|_{2}, \\
-\left(v_{R} \cdot \nabla \tilde{u}, \varphi\right) & \leq\|\nabla \tilde{u}\|_{3 / 2}\left\|v_{R}\right\|_{6}\|\varphi\|_{6} \\
& \leq C(\Sigma, T)\left(\|\xi\|_{H^{1}(0, T)}+\|\omega\|_{H^{1}(0, T)}\right)\left\|\nabla v_{R}\right\|_{2}\|\nabla \varphi\|_{2}, \\
-\left(\tilde{u} \cdot \nabla v_{R}, \varphi\right) & \leq\|\tilde{u}\|_{3}\left\|\nabla v_{R}\right\|_{2}\|\varphi\|_{6} \\
& \leq C(\Sigma, T)\left(\|\xi\|_{H^{1}(0, T)}+\|\omega\|_{H^{1}(0, T)}\right)\left\|\nabla v_{R}\right\|_{2}\|\nabla \varphi\|_{2}, \\
-\left(\omega \times v_{R k}, \varphi\right) & \leq|\omega|\|v\|_{2}\|\varphi\|_{2} \leq C(\Sigma, T) R_{0}^{2}\|\omega\|_{H^{1}(0, T)}\left\|\nabla v_{R}\right\|_{2}\|\nabla \varphi\|_{2} .
\end{aligned}
$$

The lemma follows from these inequalities and from (3-18), (3-17) and (3-4). 
Lemma 3.4. There exists a field $v$ and a sequence $\left\{v_{\rho}: \rho \in \mathscr{Y}^{\prime} \subset \mathscr{Y}\right\}$ such that, for all $R_{0}>\delta(\mathscr{B})$,

$$
\begin{aligned}
& v \in L^{2}(0, T ; V(\Omega)) \cap L^{2}\left(0, T ; L^{2}\left(\Omega_{R_{0}}\right)\right) \\
& v_{\rho} \rightarrow v \text { weakly in } L^{2}(0, T ; V(\Omega)),
\end{aligned}
$$$$
v_{\rho} \rightarrow v \text { strongly in } L^{2}\left(0, T ; L^{2}\left(\Omega_{R_{0}}\right)\right) \text {. }
$$

Proof. From the bound (3-17), we deduce that there is a subsequence of $\left\{v_{R}\right\}$, again denoted by $\left\{v_{R}\right\}$, and a field $v \in L^{2}(0, T ; V(\Omega))$ for which (3-19) $)_{2}$ holds. Fix $R_{0}>\delta(\mathscr{B})$ and apply Lemma 2.1 with $X_{0}=H^{1}\left(\Omega_{R_{0}}\right), X=L^{2}\left(\Omega_{R_{0}}\right)$ and $X_{1}=V^{\prime}\left(\Omega_{R_{0}}\right)$. There follows the existence of a subsequence, still denoted by $\left\{v_{R}\right\}$, satisfying conditions $(3-19)_{3}$. This latter subsequence may depend on $R_{0}$. However, covering $\Omega$, with an increasing sequence of bounded domains and using Cantor diagonalization method, we may select a subsequence $\left\{v_{\rho}\right\}$ for which the property (3-19) 3 holds for all $R_{0}$. The lemma is therefore proved.

In conclusion to this section, we shall prove that $u \equiv v+\tilde{u}$ is a periodic weak solution to (1-1). In view of Lemmas 2.2 and 3.4, we have only to show that $u$ satisfies (3-1). To this end, set $u_{\rho}=v_{\rho}-\tilde{u}$ in (3-2), where $\left\{v_{\rho}\right\}$ is the sequence of Lemma 3.4. Multiplying both sides of the resulting equation by an arbitrary $\psi \in C^{1}[0, T]$ such that $\psi(0)=\psi(T)$, integrating in time between 0 and $T$ and recalling that $u_{\rho}(0)=u_{\rho}(T)$, we obtain

$$
\int_{0}^{T}\left\{\left(u_{\rho}, \phi_{t}\right)-v\left(\nabla u_{\rho}, \nabla \phi\right)+\left((V-u) \cdot \nabla u_{\rho}, \phi\right)-\left(\omega \times u_{\rho}, \phi\right)+\langle f, \phi\rangle\right\},
$$

with $\phi=\psi \varphi$, for any fixed $\varphi \in \mathscr{D}(\Omega)$ and all sufficiently large $\rho$. We then pass to the limit $\rho \rightarrow \infty$ in this relation and use the convergence properties stated in (3-19). It is routine to show (see [Galdi 2000], for example) that $u$ satisfies (3-1), with $\Phi=\phi$. However, any $\Phi \in \mathscr{D}_{T, p}$ can be approximated, together with its first derivatives, uniformly pointwise by suitable linear combinations of such a $\phi$ [Galdi 2000], and so the proof of Theorem 3.2 is completed.

\section{Existence of Periodic Strong Solutions}

We now show that if $\Omega$ and the data are more regular and if these latter are sufficiently small, then a periodic strong solution exists.

Theorem 4.1. Let $\Omega$ be an exterior domain of $\mathbb{R}^{3}$ of class $C^{2}$. Let $\xi, \omega \in H^{1}(0, T)$ with $\xi(0)=\xi(T), \omega(0)=\omega(T)$, and $f \in L^{2}\left(0, T ; L^{2}(\Omega) \cap V^{\prime}(\Omega)\right)$. There is a positive constant $C_{0}=C_{0}(\Omega, v)$ such that if

(4-1)

$$
D_{\alpha} \equiv \alpha\|f\|_{L^{2}\left(0, T ; V^{\prime}(\Omega)\right)}+\|f\|_{L^{2}\left(0, T ; L^{2}(\Omega)\right)}+\alpha\left(\|\xi\|_{H^{1}(0, T)}+\|\omega\|_{H^{1}(0, T)}\right) \leq C_{0},
$$


where $\alpha=\max \{1,1 / T\}$, then there exists $(u, p)$ satisfying conditions $(i)$ and (ii) of Definition 3.1 and the following properties for all $R>\delta(\mathscr{B})$ :

$$
u \in C\left([0, T] ; L^{2}\left(\Omega_{R}\right)\right), \quad \frac{d u}{d t} \in L^{2}\left(0, T ; L^{2}\left(\Omega_{R}\right)\right)
$$

$$
\begin{aligned}
& \nabla u \in L^{2}\left(0, T ; H^{1}(\Omega)\right) \cap L^{\infty}\left(0, T ; L^{2}(\Omega)\right), \\
& \nabla p \in L^{2}\left(0, T ; L^{2}(\Omega)\right) .
\end{aligned}
$$

If , in particular, $\omega(t) \equiv 0$, we have, in addition,

$$
\frac{d u}{d t} \in L^{2}\left(0, T ; L^{2}(\Omega)\right) .
$$

In any case, $u(0)=u(T)$ and $(u, p)$ satisfies $(1-1)_{1}$ a.e. in $\Omega \times[0, T]$.

In order to construct such a solution we choose the basis functions $w_{R j}$ in (3-5) as the eigenfunctions of the Stokes problem:

$$
P \Delta w_{R j}=-\lambda_{R j} w_{R j} \quad w_{R j} \in V\left(\Omega_{R}\right) \cap H^{2}\left(\Omega_{R}\right) .
$$

Clearly, by the same argument of Step 1 of Section 3, we show the existence of a periodic approximating solution $v_{R k}$ to (3-5) that satisfies the estimates of Lemma 3.2. Under the assumptions (4-1), we shall now establish further suitable estimates on the first and second spatial derivatives of the approximating solutions.

Lemma 4.1. Let condition (4-1) hold. Then

$$
\left\|\nabla v_{R k}(t)\right\|_{2, \Omega_{R}}+\int_{0}^{T}\left\|D^{2} v_{R k}(\tau)\right\|_{L^{2}\left(\Omega_{R}\right)}^{2} d \tau \leq C,
$$

with $C$ depending only upon the data.

Proof. Multiplying both sides of (3-5) by $\lambda_{R k j} c_{R k j}$, summing over $j$ and taking into account (4-3), we deduce

$$
\text { (4-5) } \begin{aligned}
& \frac{1}{2} \frac{d}{d t}\left\|\nabla v_{R k}\right\|_{2}^{2}+\left\|P \Delta v_{R k}\right\|_{2}^{2} \\
& =-\left(\xi \cdot \nabla v_{R k}, P \Delta v_{R k}\right)+\left(\tilde{u} \cdot \nabla v_{R k}, P \Delta v_{R k}\right)+\left(v_{R k} \cdot \nabla \tilde{u}, P \Delta v_{R k}\right) \\
& -\left(\tilde{f}, P \Delta v_{R k}\right)+\left(v_{R k} \cdot \nabla v_{R k}, P \Delta v_{R k}\right)+\left(\left(\omega \times v_{R k}-\omega \times x \cdot \nabla v_{R k}\right), P \Delta v_{R k}\right),
\end{aligned}
$$

where all integrals are taken over $\Omega_{R}$. Using the Schwarz inequality along with Lemmas 2.2 and 2.3, we easily obtain from (4-5)

$$
\begin{aligned}
& \frac{d}{d t}\left\|\nabla v_{R k}\right\|_{2}^{2}+v\left\|P \Delta v_{R k}\right\|_{2}^{2} \\
& \quad \leq C(\Omega, v)\left(\left(\gamma+\gamma^{2}\right)\left\|\nabla v_{R k}\right\|_{2}^{2}+\left\|\nabla v_{R k}\right\|_{2}^{4}+\left\|\nabla v_{R k}\right\|_{2}^{6}\right)+C(v)\|\tilde{f}\|_{2}^{2},
\end{aligned}
$$


where $\gamma=\|\xi\|_{H^{1}(0, T)}+\|\omega\|_{H^{1}(0, T)}$. In order to obtain the desired estimate from this differential inequality, we need an upper bound for the quantity $\left\|\nabla v_{R k}(0)\right\|_{2}$. It is clear that

$$
\left\|\nabla v_{R k}(0)\right\|_{2}=\left\|\nabla v_{R k}(T)\right\|_{2} .
$$

From (3-15) and the mean value theorem for continuous functions, we deduce the existence of $\bar{t} \in[0, T]$ such that

$$
\left\|\nabla v_{R k}(\bar{t})\right\|_{2}^{2}=\frac{1}{T} \int_{0}^{T}\left\|\nabla v_{R k}(\tau)\right\|_{2}^{2} d \tau \leq \frac{C(\nu)}{T} \int_{0}^{T}\|\tilde{f}(\tau)\|_{-1}^{2} d \tau .
$$

Hence, from (4-6), we deduce that there exists a positive constant $C^{\prime}=C^{\prime}(\Omega, v)$ such that $D_{\alpha} \leq C^{\prime}$ implies $\left\|\nabla v_{R k}(t)\right\|_{2} \leq C\left(v, \Omega, D_{\alpha}\right)$ for all $t \in[\bar{t}, T]$. Taking into account (4-7), we then get

$$
\left\|\nabla v_{R k}(0)\right\|_{2} \leq C\left(v, \Omega, D_{\alpha}\right)
$$

and again, making suitable assumptions about the smallness of $D_{\alpha}$, we conclude

$$
\left\|\nabla v_{R k}(t)\right\|_{2} \leq C\left(v, \Omega, D_{\alpha}\right) \quad \text { for all } t \in[0, T] .
$$

Having established this, we go back to (4-6) and obtain the uniform estimate

$$
\int_{0}^{T}\left\|P \Delta v_{R k}(\tau)\right\|_{2, \Omega_{R}}^{2} d \tau \leq C\left(v, \Omega, D_{\alpha}\right)
$$

and since $\left\|D^{2} v_{R k}\right\|_{2, \Omega_{R}} \leq C\left(\left\|P \Delta v_{R k}\right\|_{2, \Omega_{R}}+\left\|\nabla v_{R k}\right\|_{2, \Omega_{R}}\right)$ (see [Heywood 1980], for example), with $C$ independent of $k$ and $R$, from (4-9) we infer (4-4) 2 .

We next provide an estimate for the time derivative of $v_{R k}$.

Lemma 4.2. Suppose the assumptions of the Lemma 4.1 hold. There exists a positive constant $C$ depending on the data and on $R$ such that

$$
\int_{0}^{T}\left\|\partial_{\tau} v_{R k}(\tau)\right\|_{2, \Omega_{R}}^{2} d \tau \leq C
$$

Proof. Multiplying (3-5) by $\frac{d c_{R k j}}{d t}$ and summing over $j$ we get

$$
\begin{aligned}
& \frac{1}{2} \frac{d}{d t}\left\|\nabla v_{R k}\right\|_{2}^{2}+v\left\|\partial_{t} v_{R k}\right\|_{2}^{2} \\
& =-\left(v_{R k} \cdot \nabla v_{R k}, \partial_{t} v_{R k}\right) \Omega_{\Omega_{R}}+\left(\xi \cdot \nabla v_{R k}, \partial_{t} v_{R k}\right)_{\Omega_{R}}+\left(\omega \times x \cdot \nabla v_{R k}, \partial_{t} v_{R k}\right)_{\Omega_{R}} \\
& \quad-\left(\omega \times v_{R k}, \partial_{t} v_{R k}\right)_{\Omega_{R}}-\left(v_{R k} \cdot \nabla \tilde{u}, \partial_{t} v_{R k}\right)_{\Omega_{R}}-\left(\tilde{u} \cdot \nabla v_{R k}, \partial_{t} v_{R k}\right)_{\Omega_{R}}+\left(\tilde{f}, \partial_{t} v_{R k}\right)_{\Omega_{R}} .
\end{aligned}
$$


We have

$$
\begin{aligned}
\left|\left(\omega \times v_{R k}, \partial_{t} v_{R k}\right)_{\Omega_{R}}\right|+\mid\left(\omega \times x \cdot \nabla v_{R k},\right. & \left.\partial_{t} v_{R k}\right)_{\Omega_{R}} \mid \\
& \leq C(\Sigma, T)\|\omega\|_{H^{1}(0, T)} R\left\|\partial_{t} v_{R k}\right\|_{2}\left\|\nabla v_{R k}\right\|_{2},
\end{aligned}
$$

and the remaining terms are estimated as in the previous lemma.

Using the estimates of Lemmas 4.1 and 4.2, and proceeding as in Step 1 of Section 3, we show the existence of a $T$-periodic field $v_{R}$ such that

$$
\begin{aligned}
v_{R} & \in L^{\infty}\left(0, T ; H^{1}\left(\Omega_{R}\right)\right) \cap L^{2}\left(0, T ; H^{2}\left(\Omega_{R}\right)\right), \\
\frac{d v_{R}}{d t} & \in L^{2}\left(0, T ; L^{2}\left(\Omega_{R}\right)\right),
\end{aligned}
$$

and satisfying, in addition, the estimate

$$
\left\|\nabla v_{R}(t)\right\|_{2, \Omega_{R}}+\int_{0}^{T}\left\|D^{2} v_{R}(\tau)\right\|_{L^{2}\left(\Omega_{R}\right)}^{2} d \tau \leq C,
$$

with $C$ independent of $R$. Furthermore, $v_{R}$ satisfies the equation (3-2). Actually, by well known arguments, (3-2) and (4-11) imply the existence of a scalar field $p_{R} \in L^{2}\left(0, T ; W^{1,2}\left(\Omega_{R}\right)\right)$ such that the pair $\left(v_{R}, p_{R}\right)$ satisfies the following equations a.a. in $\Omega \times[0, T]$ :

$$
\begin{aligned}
\frac{\partial v_{R}}{\partial t} & =v \Delta v_{R}+\left(V-v_{R}\right) \cdot \nabla v_{R}-v_{R} \cdot \nabla \tilde{u}-\tilde{u} \cdot \nabla v_{R}-\omega \times v_{R}-\nabla p_{R}+\tilde{f}, \\
\nabla \cdot v_{R} & =0 .
\end{aligned}
$$

From (4-13) we can now obtain an estimate on $\partial v_{R} / \partial t$, uniformly in $R$ on any fixed compact set, for sufficiently large $R$.

Lemma 4.3. Let the assumptions of Lemma 4.1 hold, and let $R_{0}>\delta(\Re)$. The following estimates hold:

$$
\int_{0}^{T}\left\|\partial_{t} v_{R}(t)\right\|_{2, \Omega_{R_{0}}}^{2} d t \leq C_{1} \quad \text { for all } R>R_{0}, R \in \mathscr{Y},
$$

$$
\int_{0}^{T}\left\|\nabla p_{R}(t)\right\|_{2, \Omega_{R}}^{2} d t \leq C_{2} \quad \text { for all } R \in \mathscr{Y},
$$

where $C_{1}$ depends only on the data and $R_{0}$, while $C_{2}$ depends only on the data. If, in particular, $\omega(t) \equiv 0$, we have the stronger estimate

$$
\int_{0}^{T}\left\|\partial_{t} v_{R}(t)\right\|_{2, \Omega_{R}}^{2} d t \leq C,
$$

with $C$ depending only on the data. 
Proof. By the Helmholtz decomposition, we may write

$$
\tilde{f}=P \tilde{f}+\nabla \tilde{p}, \quad \tilde{p} \in L^{2}(0, T ; G(\Omega)) .
$$

Recalling that for all $v \in V\left(\Omega_{R}\right) \cap H^{2}\left(\Omega_{R}\right)$ we have, by [Galdi and Silvestre 2005, Lemma 3(i)],

$$
((\xi+\omega \times x) \cdot \nabla v-\omega \times v) \in H\left(\Omega_{R}\right),
$$

we find from (4-13) that $P_{R} \equiv p_{R}-\tilde{p}$ satisfies the following Neumann problem (in the sense of distributions):

$$
\Delta P_{R}=\nabla \cdot F \quad \text { in } \Omega_{R},
$$

$$
\frac{\partial P_{R}}{\partial n}=F \cdot n \quad \text { at } \partial \Omega_{R},
$$

where $F=-v_{R} \cdot \nabla v_{R}-v_{R} \cdot \nabla \tilde{u}-\tilde{u} \cdot \nabla v_{R}+v \Delta v_{R}$. Formally multiplying both sides of (4-17) $)_{1}$ by $P_{R}$, integrating by parts over $\Omega_{R}$ and using (4-17) $)_{2}$ we deduce

$$
\left\|\nabla P_{R}\right\|_{2, \Omega_{R}}^{2}=\left(F, \nabla P_{R}\right) .
$$

Thus, from the Schwarz inequality, from Lemma 4.1 and from the Sobolev-like inequality

(4-18) $\quad\|w\|_{\infty, \Omega_{R}} \leq C\left(\|\nabla w\|_{2, \Omega_{R}}+\|P \Delta w\|_{2, \Omega_{R}}\right), \quad w \in H_{0}^{1}\left(\Omega_{R}\right) \cap H^{2}\left(\Omega_{R}\right)$,

(for which see [Galdi 1994a]) with $C=C(\Omega)$, we deduce that

$$
\int_{0}^{T}\left\|\nabla P_{R}(t)\right\|_{2, \Omega_{R}}^{2} d t \leq C
$$

where $C$ depends only the data. Plugging this information back in (4-13) 1 and using again Lemma 4.1, (4-16) and (4-18) we show (4-14).

We next observe that the dependence of the constant $C$ on $R_{0}$ in (4-14) is due to the presence of the term $\omega \times x \cdot \nabla v_{R}-\omega \times v_{R}$ in (4-13) $)_{1}$. Therefore, if $\omega(t)=0$, for all $t \in[0, T]$, the constant $C$ becomes independent of $R_{0}$ and we obtain the stronger estimate (4-15).

The proof of Theorem 4.1 is now achieved as follows. We multiply (4-13) by $\Phi \in \mathscr{D}_{T, p}$ and integrate over $\Omega \times[0, T]$. We then let, in the resulting equation, $R \rightarrow \infty$, along a suitable sequence, and take into account (4-12), Lemma 4.3, and the embedding $H^{1}\left(0, T ; L^{2}\left(\Omega_{R_{0}}\right)\right) \subset C\left([0, T] ; L^{2}\left(\Omega_{R_{0}}\right)\right)$, for all $R_{0}>\delta(\mathscr{B})$.

\section{References}

[Adams 1975] R. A. Adams, Sobolev spaces, Academic Press, New York, 1975. MR 56 \#9247 Zbl 0314.46030 
[Farwig et al. 2004] R. Farwig, T. Hishida, and D. Müller, " $L^{q}$-theory of a singular "winding" integral operator arising from fluid dynamics", Pacific J. Math. 215:2 (2004), 297-312. MR 2005f: 20047 Zbl 1057.35028

[Galdi 1994a] G. P. Galdi, An introduction to the mathematical theory of the Navier-Stokes equations, I: Linearized steady problems, Springer Tracts in Natural Philosophy 38, Springer, New York, 1994. Revised edition, 1998. MR 95i:35216a Zbl 0949.35004

[Galdi 1994b] G. P. Galdi, An introduction to the mathematical theory of the Navier-Stokes equations, II: Nonlinear steady problems, Springer Tracts in Natural Philosophy 39, Springer, New York, 1994. Revised edition, 1998. MR 95i:35216b Zbl 0949.35005

[Galdi 2000] G. P. Galdi, "An introduction to the Navier-Stokes initial-boundary value problem", pp. 1-70 in Fundamental directions in mathematical fluid mechanics, edited by G. P. Galdi et al., Birkhäuser, Basel, 2000. MR 2002c:35207 Zbl 01574632

[Galdi 2002] G. P. Galdi, "On the motion of a rigid body in a viscous liquid: a mathematical analysis with applications”, pp. 653-791 in Handbook of mathematical fluid dynamics, vol. I, edited by S. Friedlander and D. Serre, North-Holland, Amsterdam, 2002. MR 2003j:76024 Zbl 01942878

[Galdi 2003] G. P. Galdi, "Steady flow of a Navier-Stokes fluid around a rotating obstacle", J. Elasticity 71:1-3 (2003), 1-31. MR 2005c:76030 Zbl 02073398

[Galdi and Silvestre 2002] G. P. Galdi and A. L. Silvestre, "Strong solutions to the problem of motion of a rigid body in a Navier-Stokes liquid under the action of prescribed forces and torques", pp. 121-144 in Nonlinear problems in mathematical physics and related topics, vol. I, edited by M. S. Birman, Int. Math. Ser. 1, Kluwer/Plenum, New York, 2002. MR 2003m:76041 Zbl 1046.35084

[Galdi and Silvestre 2005] G. P. Galdi and A. L. Silvestre, "Strong solutions to the Navier-Stokes equations around a rotating obstacle", Arch. Ration. Mech. Anal. 176:3 (2005), 331-350.

[Galdi and Sohr 2004] G. Galdi and H. Sohr, "Existence and uniqueness of time-periodic physically reasonable Navier-Stokes flow past a body", Arch. Rat. Mech. Anal. 172:3 (2004), 363-406. MR 2062429 Zbl 1056.76021

[Heywood 1980] J. G. Heywood, "The Navier-Stokes equations: on the existence, regularity and decay of solutions", Indiana Univ. Math. J. 29:5 (1980), 639-681. MR 81k:35131 Zbl 0494.35077

[Hishida 1999] T. Hishida, "An existence theorem for the Navier-Stokes flow in the exterior of a rotating obstacle", Arch. Rat. Mech. Anal. 150:4 (1999), 307-348. MR 2001b:76024 Zbl 0949.35106

[Kozono and Nakao 1996] H. Kozono and M. Nakao, "Periodic solutions of the Navier-Stokes equations in unbounded domains", Tohoku Math. J. (2) 48:1 (1996), 33-50. MR 96m:35252 Zbl 0860.35095

[Ladyzhenskaya 1969] O. A. Ladyzhenskaya, The mathematical theory of viscous incompressible flow, 2nd ed., Mathematics and its Applications 2, Gordon and Breach Science Publishers, New York, 1969. MR 40 \#7610 Zbl 0184.52603

[Maremonti 1991a] P. Maremonti, "Existence and stability of time-periodic solutions to the NavierStokes equations in the whole space", Nonlinearity 4:2 (1991), 503-529. MR 92d:35227 Zbl 0737. 35065

[Maremonti 1991b] P. Maremonti, "Some theorems of existence for solutions of the Navier-Stokes equations with slip boundary conditions in half-space", Ricerche di Matematica 40:1 (1991), 81135. MR 94b:35214 Zbl 0754.35110

[Maremonti and Padula 1996] P. Maremonti and M. Padula, "Existence, uniqueness and attainability of periodic solutions of the Navier-Stokes equations in exterior domains", pp. 142-182 in Краевые задачи математической физики и смежные вопроцы теории функций, 
vol. 27, Zap. Nauchn. Sem. S.-Peterburg. Otdel. Mat. Inst. Steklov. (POMI) 233, 1996. In Russian; translation in J. Math. Sci. (New York) 93:5 (1999), 719-746. MR 2000d:35182 Zbl 0930.35126

[Morimoto 1971/72] H. Morimoto, "On existence of periodic weak solutions of the Navier-Stokes equations in regions with periodically moving boundaries", J. Fac. Sci. Univ. Tokyo Sect. IA Math. 18 (1971/72), 499-524. MR 52 \#6218 Zbl 0258.35056

[Prouse 1963] G. Prouse, "Soluzioni periodiche dell'equazione di Navier-Stokes", Atti Accad. Naz. Lincei Rend. Cl. Sci. Fis. Mat. Natur. (8) 35 (1963), 443-447. MR 30 \#779 Zbl 0128.43504

[Salvi 1995] R. Salvi, "On the existence of periodic weak solutions on the Navier-Stokes equations in exterior regions with periodically moving boundaries", pp. 63-73 in Navier-Stokes equations and related nonlinear problems (Funchal, 1994), edited by A. Sequeira, Plenum, New York, 1995. MR 97a:35183 Zbl 0848.35092

[Silvestre 2004] A. L. Silvestre, "On the existence of steady flows of a Navier-Stokes liquid around a moving rigid body”, Math. Methods Appl. Sci. 27:12 (2004), 1399-1409. MR 2005f:35251 Zbl 1061.35078

[Temam 1984] R. Temam, Navier-Stokes equations, Studies in Mathematics and its Applications 2, North-Holland, Amsterdam, 1984. MR 86m:76003 Zbl 0568.35002

[Yamazaki 2000] M. Yamazaki, "The Navier-Stokes equations in the weak- $L^{n}$ space with timedependent external force", Math. Ann. 317:4 (2000), 635-675. MR 2001f:35324 Zbl 0965.35118

Received May 11, 2004.

GIOVANNI P. GALDI

Department of Mechanical ENGINEERING

630 BENEDUM HALL

UNIVERSITY OF PITTSBURGH

PitTSBURGH, PA 15261

United STATES

galdi@engr.pitt.edu

ANA L. Silvestre

Centro de Matemática e AplicaÇões

Departamento de Matemática

INSTITUTO SUPERIOR TÉCNICO

Av. Rovisco PAIS

1049-001 LISBOA

PORTUGAL

Ana.Silvestre@math.ist.utl.pt 